\title{
Descrição da dinâmica de alimentação de idosas institucionalizadas
}

\author{
Description of the feeding dynamics of \\ institutionalized elderly women
}

\author{
Francelise Pivetta Roque ${ }^{1}$, Fernanda Maria Santana Bomfim² ${ }^{2}$ Brasília Maria Chiari ${ }^{3}$
}

\begin{abstract}
RESUMO
Objetivo: Descrever a dinâmica alimentar de idosas institucionalizadas no que se refere aos aspectos clínicos de deglutição, cognitivos, comportamentais e ambientais ligados à alimentação. Métodos: Realizou-se um estudo observacional transversal prospectivo, que descreveu a dinâmica de alimentação de 30 idosas residentes numa instituição de longa permanência. Alguns dos aspectos analisados foram registrados por observadores no momento da alimentação e outros em filmagem da refeição de cada idosa. Os dados foram apresentados de forma descritiva e comparados à literatura. Resultados: A média de idade foi 83,7 anos. Os diagnósticos médicos mais prevalentes foram síndrome demencial e hipertensão arterial sistêmica. Foram observados: ingestão oral pobre em 73,3\% das idosas, tempo médio de alimentação de 10,1 minutos, posicionamento inadequado em $46,7 \%$ das refeições, alterações atitudinais em $16,7 \%$ e comportamentais em $40 \%$ das idosas. A assistência foi requerida por $56,6 \%$ das idosas, tendo sido provida a $70,6 \%$ desses casos, e julgada inadequada em $66,6 \%$ deles. Eram edêntulas $44,4 \%$ das idosas, das quais $46,1 \%$ utilizavam prótese dentária total, sendo predominante a inadequação da adaptação, do estado de conservação e da higiene $(83,4 \%)$. A prevalência de alterações de deglutição foi igual a 23,3\%, sendo as mais prevalentes os resíduos alimentares após a deglutição, tosse durante a alimentação e alteração vocal após a deglutição. Conclusão: Verificou-se presença significativa de fatores ambientais, cognitivos e comportamentais ligados à alimentação que podem oferecer risco à deglutição. Também se identificaram outras alterações específicas da deglutição, cujo gerenciamento é importante para evitar complicações da saúde destas idosas.
\end{abstract}

Descritores: Instituição de longa permanência para idosos; Alimentação; Nutrição do idoso; Transtornos de deglutição; Demência; Idoso; Mulheres; Cuidadores

\section{INTRODUÇÃO}

O envelhecimento da população mundial tem sido bastante discutido nesta última década, por se tratar de um fenômeno universal, característico tanto dos países desenvolvidos como, de modo crescente, dos países em desenvolvimento ${ }^{(1,2)}$.

A rapidez com que ele tem ocorrido no Brasil, em cuja população $10,2 \%$ são idosos ${ }^{(3)}$, integra um contexto de desigualdades sociais, economia inconsistente, elevado nível de

Trabalho realizado na Faculdade de Fonoaudiologia da Universidade Estadual de Ciências da Saúde de Alagoas - UNCISAL - Maceió (AL), Brasil.

(1) Doutora, ProfessoraAdjunto da Faculdade de Fonoaudiologia da Universidade Estadual de Ciências da Saúde de Alagoas - UNCISAL - Maceió (AL), Brasil.

(2) Acadêmica do Curso de Fonoaudiologia da Faculdade de Fonoaudiologia da Universidade Estadual de Ciências da Saúde de Alagoas - UNCISAL Maceió (AL), Brasil.

(3) Livre-docente, Professora Titular do Departamento de Fonoaudiologia da Universidade Federal de São Paulo - UNIFESP - São Paulo (SP), Brasil.

Endereço para correspondência: Francelise Pivetta Roque. R. Dr. José

Correia Filho, 282/805, Ponta Verde, Maceió (AL), Brasil, CEP: 57035-130.

E-mail: franceliseroque@yahoo.com.br

Recebido em: 19/1/2009; Aceito em: 4/8/2009 pobreza, acesso precário aos serviços de saúde e baixa renda familiar, sem as mudanças estruturais necessárias às demandas desse novo grupo etário ${ }^{(4)}$.

$\mathrm{O}$ avanço da idade propicia o estabelecimento de doenças, incapacidades e dependência, que são experimentadas em grande parte pelas mulheres idosas. Estudos demonstram que elas vivem mais que os homens, são mais sozinhas (pela maior dificuldade para se casarem novamente), apresentam menores níveis de instrução formal e renda e maior frequência de queixas de saúde ${ }^{(5,6)}$. Estes fatores incrementam as probabilidades de elas dependerem de instituições de suporte, como as chamadas instituições de longa permanência para idosos (ILPI), comumente chamadas de "asilos", ou "casas de respouso", cujo perfil comum no Brasil é a de residentes com graus de dependência acentuados, com comprometimento da independência e autonomia ${ }^{(7,8)}$.

Nas ILPIs, são frequentes os problemas alimentares, que aumentam a responsabilidade nos cuidados com estes idosos, frequentemente acometidos por outras incapacidades, sendo a pneumonia aspirativa uma das principais causas de morbi-mortalidade nesta população, associada à internação 
constante e de longa estadia, tornando a assistência onerosa para a instituição ${ }^{(9,10)}$.

Estes problemas alimentares incluem os sinais disfágicos, mas não se limitam a eles, e sua prevalência foi descrita como $26,5 \%$ a $87 \%$ dos idosos institucionalizados, conforme estudos norte-americanos e europeus. Estes distúrbios incluem sinais de disfagia, dependência para alimentação, alterações de comportamento interferentes na alimentação, ingestão reduzida (por várias razões), consistência inadequada do alimento, oferta rápida do alimento pelo cuidadora, problemas relacionados à postura e posicionamento, perda dentária ou outras alterações dentárias que levem à dor ${ }^{(11-13)}$.

Considerando-se o grande impacto destes problemas no estado de saúde de idosos institucionalizados, conhecer os aspectos nela envolvidos é essencial para identificar estes problemas, a fim de determinar intervenções mais apropriadas, tanto fonoaudiológicas como interdisciplinares, não somente para os idosos residentes, em termos individuais, como em termos coletivos, a exemplo dos treinamentos voltados aos cuidadores.

Tendo-se em vista que os aspectos responsáveis por estes problemas alimentares podem variar de acordo com fatores próprios de cada localidade, considerando-se o âmbito sócioeconômico-cultural no qual a saúde está inserida, tornam-se necessárias pesquisas brasileiras neste tema.

Desta forma, este estudo objetiva descrever a dinâmica de alimentação de idosas institucionalizadas, no que diz respeito aos aspectos clínicos de deglutição, cognitivos, comportamentais e ambientais ligados à alimentação.

\section{MÉTODOS}

O projeto desta pesquisa foi analisado e aprovado pelo Comitê de Ética em Pesquisa da Universidade Estadual de Ciências da Saúde de Alagoas (UNCISAL), com protocolo número 788. Todos os sujeitos envolvidos e/ou seus tutores legais assinaram o Termo de Consentimento Livre e Esclarecido, consentindo a realização e divulgação deste estudo, conforme Resolução 196/96 (BRASIL. Resolução MS/CNS/ CNEP nº 196/96 de 10 de outubro de 1996).

Trata-se de um estudo observacional transversal prospectivo, que descreve a dinâmica de alimentação de 30 das 35 pessoas residentes em uma instituição de longa permanência para idosos (ILPI) em Maceió, Alagoas, na qual só habitam mulheres. Uma idosa foi excluída do estudo por ter falecido antes que seus dados fossem coletados, e quatro residentes não participaram por não terem, na época da coleta dos dados, a idade mínima para serem consideradas idosas. Também foi critério de exclusão a utilização exclusiva de via alternativa de alimentação à época do estudo (sonda nasogástrica, nasoenteral, ou ostomia), porém nenhum dos residentes preenchia este quesito.

A instituição estudada se enquadra na definição de instituições de longa permanência para idosos (ILPI) proposta pela ANVISA ${ }^{(14)}$, que as conceitua como instituições governamentais ou não governamentais, de caráter residencial, destinadas a domicilio coletivo de pessoas com idade igual ou superior a 60 anos, com ou sem suporte familiar, em condição de liberdade, dignidade e cidadania.
A ILPI onde o estudo foi realizado foi escolhida por ser a única na referida capital que era assistida por uma equipe interdisciplinar especializada em Saúde do Idoso, e estudantes de graduação, ligados a uma Universidade Pública, das áreas de Medicina, Fonoaudiologia, Fisioterapia, Terapia Ocupacional, Serviço Social, Nutrição, Psicologia e Enfermagem, buscandose eliminar, desta forma, vieses de pesquisa decorrentes de um acompanhamento profissional em saúde insuficiente ou inadequado. Estas idosas recebiam assistência de cuidadoras formais, pagas pela instituição, ou pela própria idosa ou sua família. Todas haviam passado por um treinamento de cuidadores inicial, com conceitos básicos e suas diferenças de escolaridade e de conhecimento, verificadas na ocasião deste treinamento, não se associavam ao fato de elas serem pagas pela ILPI ou pela família/idosa, tendo algumas delas também já sido orientadas de forma individual pelos profissionais atuantes nesta ILPI.

Os dados foram coletados mediante análise do prontuário, e observação de uma refeição (almoço) de cada idosa.

Foram coletados no prontuário os dados de identificação (idade, estado civil e escolaridade), tempo de institucionalização, diagnósticos médicos, medicações utilizadas (número e categorias), e grau de independência nas atividades básicas de vida diária, medida pelo índice de Katz ${ }^{(15)}$.

Os dados da dinâmica de alimentação foram obtidos de duas formas: em tempo real, no momento imediato que antecedia a refeição, no seu início e ao final da mesma; e mediante análise posterior do registro audiovisual (filmagem) feito de cada refeição. Em tempo real da alimentação, os procedimentos necessários à obtenção dos dados (apresentados a seguir, na descrição do protocolo utilizado) variou de acordo com o item analisado, incluindo a inspeção da cavidade oral, a observação do ambiente (físico e humano) em que se dava a alimentação e a observação da idosa (geral e específica em relação a alguns itens de deglutição, como, por exemplo, do ritmo respiratório durante a mesma, e da qualidade vocal após a deglutição em comparação à anterior à refeição).

As filmagens foram realizadas por dois estudantes de graduação em Fonoaudiologia treinados para tal procedimento, e serviram ao registro dos itens "aspectos atitudinais e comportamentais relacionados à alimentação" e "dependência alimentar e assistência requerida/provida", realizados por uma fonoaudióloga e gerontóloga, com seis anos de experiência no atendimento a idosos com alterações de deglutição. Os demais itens, registrados no momento real da alimentação ou próximo a esta, foram obtidos por um dos dois estudantes responsáveis pela filmagem. Estes observadores foram treinados para a realização destes registros, tendo sido julgados aptos para tal após a coincidência em cinco registros seguidos de cada item avaliado em outros idosos, não inseridos neste estudo.

Cada refeição foi observada no seu local habitual, utilizandose, para a filmagem, uma câmera digital Sony® DSC-T10 com resolução máxima de 640x480 pixels e tripé compatível, montados a uma distância que possibilitou a visualização da idosa e, quando pertinente, da sua cuidadora. Ambas foram instruídas a agir naturalmente durante toda a filmagem, cuja duração correspondeu à refeição completa. O pesquisador responsável pela filmagem somente esteve presente no início e após o final 
desta, para que os sujeitos pudessem usufruir a maior parte do tempo a sós, como acontece na alimentação habitual. Tais condições de observação visaram a preservar a naturalidade do momento, a fim de que elas fossem as mais fidedignas possíveis da ocorrência habitual dos fenômenos estudados.

O protocolo utilizado para registro dos dados foi desenvolvido a partir dos itens que compunham instrumento utilizado em estudo anterior $^{(12)}$. Na presente pesquisaforam feitos acréscimos ou subtrações, resultando num protocolo composto por questões de múltipla escolha. A seguir são apresentados os itens que compuseram tal questionário, bem como as respostas possíveis e os critérios de análise adotados para cada resposta.

- Características da refeição/dieta. Este item foi avaliado quanto a: refeição observada (almoço/jantar), consistência alimentar, viscosidade do líquido, quantidade ingerida e tempo total gasto para se alimentar (em minutos). Foram critérios de análise para a quantidade ingerida sua caracterização visual, tendo como referência a quantia ofertada (total). Assim, a quantia ingerida foi classificada como"metade" do total ofertado, "três quartos do total", "tudo" ou "menos que a metade". O tempo total gasto foi calculado considerando-se o início como o primeiro movimento manual que levou o alimento à boca (tendo sido este movimento da idosa ou da cuidadora) até a finalização da refeição;

- Ambiente de alimentação. Este item foi avaliado quanto ao local geral de alimentação da idosa (quarto, refeitório e outro), local específico de alimentação da idosa (cadeira, cadeira de rodas, cama ou outro), número pessoas no local da refeição, características do ambiente (luminosidade; ambiente sonoro; distratores visuais), adequação do posicionamento e problemas específicos dele;

- Aspectos atitudinais e comportamentais relacionados à alimentação. Neste item foram considerados a atitude em relação à alimentação, eventuais problemas de comportamento durante a refeição, a inadequação da quantidade de alimento ou líquido colocado pela idosa à boca por vez, a inadequação da velocidade com que o alimento ou líquido é colocado pela idosa à boca por vez. A avaliação destes itens foi subjetiva, conforme o que se observa em estudos anteriores (incluindo-se a velocidade com que o alimento é colocado à boca da idosa, para a qual não existem, na literatura, parâmetros objetivos) tendo-se em vista, entretanto, os conceitos que embasaram cada uma das categorias. As alterações atitudinais foram consideradas como algum desvio da conduta da idosa frente ao alimento, relacionadas à aceitação do mesmo, podendo esta estar presente, porém desviada (ex.: tentativa de ingestão de objetos ou outros que não alimento) ou diminuída (ex.: rejeição do alimento ou demonstração de desinteresse pelo mesmo). As alterações de comportamento foram consideradas como qualquer comportamento que interferisse na alimentação, podendo este ser alteração da atenção, agressividade, ato de falar, gritar ou cantar durante a alimentação, sonolência, agitação psicomotora, dentre outras. A quantia de alimento colocada por vez à boca foi analisada segundo o que se considera comumente aceitável na cultura brasileira, sendo registrados os extremos passíveis de observação: muito menos do que o utensílio comportava ou resultando em volume nas bochechas e dificuldade para gerenciar o alimento pelo aumento da quantia colocada, podendo inclusive ocasionar escape extra-oral do mesmo. Em relação à velocidade de ingestão, novamente se considerou o comumente aceitável, registrando-se extremos que chamassem a atenção: lentidão exagerada, ou aumento da velocidade.

- Dependência alimentar e assistência requerida/provida: assistência requerida para se alimentar, assistência provida para se alimentar, tipo de assistência provida;

- Aspectos bucais: dentição natural (dentes ausentes, estado de conservação e higiene), prótese dentária (uso, adaptação, estado de conservação e higiene);

- Problemas de deglutição. Este item do questionário foi composto pelos seguintes aspectos: escape oral anterior (escorrimento do alimento ou líquido pelos lábios, após a captação do bolo); resíduos alimentares após a deglutição (acúmulo de alimento em vestíbulo anterior, lateral, assoalho bucal e/ou superfície lingual após a deglutição); alteração vocal após a deglutição (voz molhada - som borbulhante na voz, ou qualquer outra mudança na qualidade vocal após a deglutição, indicativo de estase de secreções, líquidos ou alimentos no vestíbulo laríngeo); fase oral aumentada (tempo entre a captação completa do bolo até o início da elevação do complexo hiolaríngeo, determinada pelo disparo do reflexo de deglutição, superior ao comumente esperado); comportamento de cuspir o alimento ou a saliva; comportamento de morder o utensílio; movimento de língua antagônico à entrada de alimento; tosse durante a alimentação (resposta reflexa de proteção da via aérea contra a entrada de corpos estranhos, realizada pelo indivíduo sem solicitação), engasgo (obstrução do fluxo aéreo, parcial ou completo, decorrente da entrada de um corpo estranho nas vias aéreas inferiores); fadiga/alteração respiratória (coloração azulada da pele, causada por pressões excessivas de hemoglobina desoxigenada nos plexos capilares e venosos e/ou broncoespasmo;

- Dificuldade respiratória causada por uma constrição repentina dos músculos das paredes brônquicas, alteração na frequência respiratória, na ocorrência de queda ou aumento excessivo da frequência respiratória, tendo como base a faixa de normalidade de 12 a 20 respirações por minuto); refluxo oral de alimento ou líquido (retorno do material já deglutido à cavidade oral); refluxo nasal de alimento ou líquido (desvio do material inserido na cavidade oral para a cavidade nasal); movimentação compensatória para deglutir ${ }^{(16)}$.

Os dados foram apresentados de forma descritiva, utilizando-se frequência absoluta, relativa, média, variância e desvio-padrão, e comparados à literatura. Cada aspecto da dinâmica da alimentação também foi analisado em relação a diagnósticos médicos da idosa que pudessem justificar dificuldade de origem motora ou cognitiva, e em relação à dependência alimentar, demonstrada em estudos anteriores como preditora de dificuldades de deglutição. 


\section{RESULTADOS}

A média de idade da população estudada foi de $83,73 \pm 10,7$ anos (idade mínima de 62 e máxima de 106 anos), 70\% na faixa igual ou superior a 80 anos.

No que se refere à escolaridade, 56,6\% eram analfabetas, $13,3 \%$ tinham até quatro anos de estudo, $23,3 \%$ haviam estudado de quatroa oito anos, e 6,6\%, mais do que oito anos. Em relação ao estado civil, uma era casada, 15 eram solteiras, e 14, viúvas.

Quanto ao tempo de institucionalização, uma idosa residia na ILPI há menos de dois anos; 12 idosas (40\% da população) residiam entre dois e cinco anos; dez idosas $(33,3 \%)$, entre cinco e dez anos; e sete idosas (23,3\%), estavam institucionalizadas há mais de dez anos.

A distribuição dos diagnósticos médicos das idosas é apresentada na Tabela 1. Em relação ao número de diagnósticos, 13,2\% apresentavam apenas um único diagnóstico, sendo as demais acometidas por duas ou mais comorbidades: $36,6 \%$ das idosas tinham dois diagnósticos associados, $36,6 \%$ das idosas tinham três diagnósticos e $13,2 \%$ das idosas, quatro diagnósticos.

Tabela 1. Distribuição de idosas segundo os tipos de diagnósticos médicos

\begin{tabular}{lc}
\hline Diagnósticos médicos & Prevalência \\
& $\mathrm{N}(\%)$ \\
\hline Síndrome demencial & $15(50)$ \\
Hipertensão arterial sistêmica & $14(46,6)$ \\
Amaurose & $6(19,8)$ \\
Diabetes & $5(16,6)$ \\
Acidente Vascular Encefálico (AVE) & $4(13,2)$ \\
Dislipidemia & $4(13,2)$ \\
Depressão & $3(9,9)$ \\
Déficit auditivo & $3(9,9)$ \\
Osteoartrite & $2(6,7)$ \\
Desnutrição & $2(6,7)$ \\
Insuficiência venosa periférica & $2(6,7)$ \\
Hipotiroidismo & $2(6,7)$ \\
Transtorno psicótico & $2(6,7)$ \\
Deficiência visual, & $1(3,3)$ \\
Câncer & $1(3,3)$ \\
Epilepsia & $1(3,3)$ \\
Insuficiência cardíaca congestiva & $1(3,3)$ \\
\hline
\end{tabular}

O uso de medicamentos foi indicado para $83,3 \%$ do total, sendo que $56 \%$ destas usavam pelo menos quatro. Dentre as medicações mais utilizadas pelas idosas, $53 \%$ utilizavam medicações neuropsiquiátricas, incluindo benzodiazepínicos, anticolinérgicos, antidepressivos, antipsicóticos e neurolépticos, e 53\% utilizavam anti-hipertensivos.

De acordo com a avaliação da dependência para atividades da vida diária ${ }^{(15)}$ atribuída às idosas, aproximadamente $33 \%$ apresentou-se independente para qualquer atividade, $30 \% \mathrm{com}$ algum grau de dependência e $36,7 \%$, totalmente dependentes. Dentre estas últimas, 63,6\% tinham demência e 27,3\% apresentaram diagnóstico de acidente vascular encefálico (AVE).

Quanto à dinâmica de alimentação, no que se refere às características da refeição e dieta, a consistência semi-sólida foi ofertada a metade da população total, e a $42,8 \%$ das idosas edêntulas que não faziam uso de prótese dentária. A outra metade recebeu dieta modificada (branda em $20 \%$ delas, pastosa em $10 \%$ e pastosa fina em $20 \%$ ). Todas as idosas receberam também líquido ralo na refeição.

A ingestão de três quartos ou mais da refeição foi observada em $73,3 \%$ das idosas. A ingestão oral pobre (metade ou menos da quantia) foi verificada em $26,7 \%$ da população, tendo havido duas idosas $(6,7 \%)$ que ingeriram um quarto ou menos. Todas as idosas com ingestão oral pobre necessitavam de assistência para se alimentar, embora somente metade destas tenha recebido este auxílio. Sessenta e cinco por cento das idosas com ingestão oral pobreeram demenciadas.

O tempo médio de duração das refeições foi de 10,1 $1 \pm 5,7$ minutos, com tempo mínimo de 2,76 e máximo de 22,03 minutos. Dentre as idosas $53,3 \%$ demoraram até dez minutos para se alimentar. Destas, $75 \%$ apresentavam diagnóstico de demência sendo $50 \%$ dependentes para alimentação (dependência requerida e provida).

Em relação ao ambiente de refeição, 14 idosas se alimentaram no refeitório, 14 no quarto e duas na sala de convivência.

A Tabela 2 apresenta o local em que as idosas se alimentavam, sendo que, dentre as que o faziam na cama, $41,6 \%$ apresentavam inadequação de posicionamento. "Cabeça anteriorizada" foi o aspecto mais comumente alterado, seguido por "escorrega para um lado" e "apoio insuficiente".

Tabela 2. Distribuição de idosas segundo local de alimentação

\begin{tabular}{lc}
\hline Local de alimentação & Prevalência \\
& $\mathrm{N}(\%)$ \\
\hline Cadeira & $16(53)$ \\
Cadeira de rodas & $2(6,7)$ \\
Cama & $12(40)$ \\
Total & $30(100)$ \\
\hline
\end{tabular}

Em relação à assistência requerida para alimentação, ela foi evidenciada em aproximadamente 17 idosas $(56,6 \%)$, tendo 12 delas $(70,5 \%)$ o diagnóstico de demência, quatro $(23,5 \%)$ idosas o de AVE, e uma o de transtorno psiquiátrico. A assistência requerida foi provida a 70,6\% das idosas, sendo julgada como inadequada em $66,6 \%$ das que a receberam. Em $75 \%$ dos casos de inadequação verificou-se aumento da quantidade ofertada por vez, seguido pela ausência ou diminuição de pistas necessárias (50\%) e pelo aumento da velocidade de oferta $(37,5 \%)$.

As inadequações ambientais para a refeição são apresentadas na Tabela 3. A maioria das idosas com problema de posicionamento se encontrava no quarto (55\%), seguida pelo refeitório (33\%). Todas elas eram dependentes na alimentação, embora metade não tenha recebido a assistência, e em $77,8 \%$ foi feito o diagnóstico de demência. Entre as idosas que comeram em ambiente com má iluminação, todas possuíam demência e dependência (requerida e provida), e entre as idosas que se alimentaram em ambiente ruidoso, oito $(44,5 \%)$ tinham diagnóstico de demência, e 50\% requeriam assistência para alimentação. Entre as idosas em cujo local havia distratores 
visuais, 46,7\% possuíam diagnóstico de demência, e 46,7\% eram dependentes para a alimentação.

Tabela 3. Distribuição das idosas quanto a inadequações ambientais

\begin{tabular}{lc}
\hline Inadequação no ambiente de alimentação & $\begin{array}{c}\text { Prevalência } \\
\mathrm{N}(\%)\end{array}$ \\
\hline Posicionamento inadequado & $14(46,7)$ \\
Ambiente com má iluminação & $5(16,7)$ \\
Ambiente barulhento & $18(60)$ \\
Presença de distratores visuais & $15(50)$ \\
\hline
\end{tabular}

Na Tabela 4 pode-se observar as inadequações de atitudes e comportamentos de idosas durante a refeição. Constatou-se que $16,7 \%$ apresentaram comportamentos não-colaborativo. As duas idosas que rejeitaram o alimento apresentavam diagnóstico de demência e dependência para alimentação; todas as três que demonstraram desinteresse requeriam assistência para se alimentar, tendo duas delas recebido esta assistência. Os diagnósticos médicos dentre estas foram distintos (transtorno psiquiátrico, AVE e demência).

Tabela 4. Distribuição das idosas com inadequações atitudinais e comportamentais

\begin{tabular}{lc}
\hline Inadequações atitudinais e comportamentais & $\begin{array}{c}\text { Prevalência } \\
\mathrm{N}(\%)\end{array}$ \\
\hline $\begin{array}{l}\text { Atitude não-colaborativa } \\
\text { Rejeição }\end{array}$ & $2(6,7)$ \\
$\quad$ Desinteresse & $3(10)$ \\
Alterações comportamentais & \\
$\begin{array}{l}\text { Distratibilidade } \\
\text { Fala, grita ou canta durante a alimentação }\end{array}$ & $7(23,3)$ \\
$\quad$ Sonolência & $1(13,3)$ \\
Aumento na quantia de alimento ou líquido \\
colocada à boca por vez durante a auto- \\
alimentação \\
$\begin{array}{l}\text { Aumento da velocidade de ingestão na auto- } \\
\text { alimentação }\end{array}$ \\
\hline
\end{tabular}

Em relação aos aspectos bucais, de todas as idosas avaliadas, pode-se verificar que $13(44,4 \%)$ idosas eram totalmente edêntulas, das quais seis $(46,1 \%)$ utilizavam prótese dentária total (superior e inferior em uma idosa e, emcinco delas, apenas superior). Em 83,4\% das usuárias de prótese dentárias, estas estavam inadequadas quanto ao estado de conservação. Dentre as idosas com dentição presente (17 idosas), 94,1\% apresentaram perda de mais de 15 dentes, estado de conservação dentária e higiene oral inadequados. Nove destas 17 idosas $(52,9 \%)$ tinham diagnóstico de demência, e todas elas requeriam assistência para se alimentar.

A prevalência das alterações de deglutição foi de 23,3\%, conforme descrito naTabela 5, tendo sido as mais observadas: resíduos alimentares após a deglutição, tosse durante a alimentação e alteração vocal após a deglutição. Os diagnósticos de demência e AVE estiveram frequentemente associados a estas alterações de deglutição, bem como a assistência requerida para se alimentar. Não se verificaram refluxos orais ou nasais de alimento, dificuldade para abrir a boca, ato de morder o utensílio, nem movimento de língua antagônico à entrada de alimento.

Tabela 5. Distribuição das idosas por alteração da deglutição

\begin{tabular}{lc}
\hline Alteração de deglutição & $\begin{array}{c}\text { Prevalência } \\
\mathrm{N}(\%)\end{array}$ \\
\hline Resíduos alimentares após a deglutição & $7(23,3)$ \\
Tosse durante a alimentação & $6(20)$ \\
Alteração vocal após a deglutição & $5(16,7)$ \\
Fadiga/alteração respiratória & $3(10)$ \\
Fase oral aumentada & $3(10)$ \\
Ato de cuspir o alimento & $2(6,7)$ \\
Engasgo & $2(6,7)$ \\
Vedamento labial inadequado & $2(6,7)$ \\
\hline
\end{tabular}

\section{DISCUSSÃO}

Apesar de este estudo não ter avaliado a dinâmica de alimentação nos homens, pela característica da instituição pesquisada, estudos brasileiros realizados com idosos institucionalizados demonstram que os homens raramente representam mais do que $20 \%$ do total dos residentes em ILPIs ${ }^{(4,17)}$. Essa tendência também verificada em outros países, como Canadá, Israel e Estados Unidos, nos quais a proporção de mulheres dentre os idosos institucionalizados aumenta de acordo com idade ${ }^{(18)}$.

A idade avançada, o tempo de escolaridade reduzido e o estado civil solteiro ou viúvo, correspondentes à maioria das idosas estudadas, refletem os dados brasileiros ${ }^{(3,5)}$, e estão de acordo com a literatura nacional e internacional acerca do perfil encontrado em ILPIs ${ }^{(7,9)}$. Demonstram ainda a susceptibilidade destes indivíduos ao estabelecimento de morbidades e dependência, que comprometem diversas atividades da vida diária (AVDs), incluindo a alimentação ${ }^{(19)}$, visto que o aumento da prevalência de alterações da condição física e cognitiva a cada ano de idade é maior dentre os considerados "muito idosos". $\mathrm{O}$ aumento da idade se mostrou como fator de risco para pneumonia, sobretudo a aspirativa, devido à maior propensão à infecção por bactérias gram-negativas ${ }^{(11,20)}$.

Neste estudo verificou-se uma elevada prevalência de co-morbidades, fato comumente referido em idosos, especialmente quando institucionalizados, e apontado como fator preditivo para disfagia e pneumonia aspirativa ${ }^{(9,21)}$.

A demência, encontrada em metade das idosas estudadas, é uma das principais responsáveis por alterações da deglutição desde a sua fase inicial, em princípio comprometendo os aspectos da fase pré-oral, que envolvem os fatores comportamentais e a cognitivos relacionados à alimentação ${ }^{(22)}$. Conforme descrito nos resultados, grande parte das idosas do presente estudo alimentadas em condições desfavoráveis, tanto ambientais (iluminação, sonoridade, distratores visuais, posicionamento no local específico de alimentação) quanto falta de assistência dos cuidadores, apresentavam diagnóstico de demência, demonstrando o aumento da predisposição das mesmas a alterações de deglutição. 
Os tipos de medicação mais utilizados pelas idosas desta pesquisa, neuropsiquiátricos e anti-hipertensivos, corresponderam em parte aos apontados em estudo recente que descreveu o perfil dos residentes de ILPIs ${ }^{(23)}$, nos quais houve o uso predominante de anti-hipertensivos, diuréticos e hipoglicemiantes orais. Os medicamentos predominantes na população estudada são referidos pela literatura como produtores potenciais de efeitos adversos que podem interferir no mecanismo normal de deglutição, podendo gerar disfagia, pelo fato de terem como efeitos colaterais a depressão da atividade do sistema nervoso central, a redução do tônus da musculatura dos órgãos fonoarticulatórios, a diminuição do estado de alerta do indivíduo e a produção de xerostomia, favorecendo a perda dentária, gengivite, o crescimento de bactérias patógenas ao pulmão se aspiradas, e a redução do nível de alerta ${ }^{(21)}$.

O número elevado de medicamentos utilizados pelas idosas estudadas está de acordo com a literatura, e assinala um risco maior para o desenvolvimento de disfagia e pneumonia aspirativa $^{(9)}$. $\mathrm{O}$ aumento do número de prescrições, além de predispor as idosas às dificuldades de deglutição, é também condição de uma pessoa doente, que, portanto, pode estar com a função imune comprometida.

Percebe-se que a população estudada reflete o perfil de fragilidade encontrado em ILPI, associado à impossibilidade, indisponibilidade, desconhecimento dos familiares para o cuidado e à necessidade de internação.

Conforme descrito nos resultados, o diagnóstico de demência e dependência alimentar determinaram a quase totalidade das consistências alimentares modificadas. Este dado está de acordo com estudo anterior, em que a dependência alimentar se mostrou diretamente relacionada à alteração cognitiva ${ }^{(11)}$.

Em relação ao local de alimentação, verificou-se que a proporção de idosas que se alimenta no refeitório e no quarto é semelhante. Neste último, ocorre grande parte dos problemas de posicionamento, em paralelo à ausência de condições para adequação, iluminação imprópria e apoio insuficiente para os utensílios utilizados nesta atividade. Já o refeitório, por ser local exclusivo para o momento da alimentação, pode favorecer a mesma, embora também sejam necessárias adaptações, não somente do local, tendo-se em vista que todas as refeições registradas no refeitório demonstraram presença de distratores visuais e barulho intenso, que podem interferir de forma negativa na alimentação, como também do número de residentes a serem alimentados.

O percentual de assistência requerida pelas idosas desta pesquisa se aproxima do valor documentado na literatura ${ }^{(12,24)}$, demonstrando que aproximadamente $50 \%$ dos residentes de ILPIs requereram assistência para se alimentar. Constatou-se ainda que nem todas as idosas foram auxiliadas de forma apropriada na alimentação, havendo altos índices de inadequação do posicionamento no local de refeição, de fornecimento de pistas para alimentação, do controle do volume e da velocidade da ingestão, o que pode repercutir negativamente na dinâmica alimentar das mesmas. A dependência das idosas para alimentação provavelmente aumenta o risco de pneumonia aspirativa se os cuidadores não estiverem apropriadamente informados e treinados, especialmente quando os pacientes têm disfagia ou estado cognitivo reduzido. Eles podem ofertar alimentos e líquidos rapidamente e em quantidade excessiva, propiciando a broncoaspiração ${ }^{(9)}$.

O tempo requerido para alimentação menor que dez minutos foi verificado em mais da metade da população estudada, sendo a maioria composta por idosas com demência e dependentes para alimentação. O tempo reduzido, associado ao déficit cognitivo e dependência podem repercutir tanto na diminuição da ingestão, identificada em mais de um quarto das idosas, como na recusa alimentar, identificada em 16,7\%, bem como nas alterações de deglutição verificadas. Estudo anterior demonstrou que o tempo de 35 a 40 minutos para fornecer auxílio de alimentação melhorou a ingestão de alimento e consumo de líquido, independentemente do nível de dependência para comer ${ }^{(25)}$. Idosos incapazes de se alimentar sozinhos têm suas refeições frequentemente ofertadas por cuidadores ou membros da família que pelo desconhecimento dos problemas de deglutição, ofertam o alimento no menor tempo possível, o que pode ser equivalente a forçar a alimentação ${ }^{(26)}$.

Os resultados desta pesquisa estão de acordo com os de estudo anterior ${ }^{(12)}$, no que se refere à prevalência de inadequações posturais para alimentação associada a dificuldades cognitivas. Na maioria dos casos se tratava de idosas com diagnóstico de demência, necessitadas de assistência alimentar, nem sempre disponível na instituição.

Embora a má iluminação no ambiente de alimentação não tenha sido frequente, em todos os casos em que isto ocorreu tratava-se de idosa com demência e dependência alimentar. O fato de todas as idosas alimentadas em ambientes com iluminação inadequada serem portadoras de demência e dependentes, pode indicar a falta de preocupação ou desconhecimento do cuidador sobre o impacto das alterações no nível de atenção e acuidade visual na dinâmica de alimentação.

No que diz respeito aos distratores visuais e à poluição sonora, estes acompanharam a maior parte das idosas, mas não se limitaram àquelas com alterações cognitivas, e foram frequentes no refeitório, onde habitualmente havia em média dez pessoas presentes no ambiente. Apesar de não existirem estudos sobre as condições de luminosidade, ambiente sonoro e distratores visuais durante a alimentação de idosos institucionalizados, sabe-se da relevância destes fatores para a fase pré-oral da deglutição ${ }^{(27)}$, especialmente quando existe algum comprometimento cognitivo.

As alterações atitudinais e de comportamento encontradas nesta população foram superiores aos estudos anteriores que expõem a prevalência de $23 \%$, porém estão de acordo com os mesmos no que diz respeito à sua relação com alterações cognitivas e dependência alimentar ${ }^{(12,26)}$. O fornecimento de pistas visuais e auditivas, e a oferta adequada da dieta (consistência, volume e velocidade) têm efeito positivo no gerenciamento de alterações atitudinais e comportamentais, favorecendo a alimentação.

A perda dentária foi representativa na população estudada, visto que aproximadamente metade era totalmente edêntula, e das parcialmente edêntulas, 94,1\% delas havia perdido mais de 15 dentes. Em ambos os grupos, as idosas com demência representavam mais de $50 \%$ das que requeriam assistência para se alimentar. A dentição pobre é um dos aspectos mais frequentemente interferentes na alimentação $^{(24)}$, havendo 
correlação entre o edentulismo e a ocorrência de disfagia e pneumonia aspirativa ${ }^{(9)}$.

Quanto ao uso de prótese, observou-se uma prevalência elevada $(83,4 \%)$ das que estavam mal adaptadas, com higiene e estado de conservação inadequados, superior aos dados representados pela literatura, em que o mau estado de conservação foi encontrado em $68 \%$ dos residentes da ILPI estudada ${ }^{(12)}$, o que pode refletir as diferenças sócioeconômico-culturais entre os grupos estudados, já que no Brasil a saúde bucal da população é precária. Embora o uso de próteses dentárias por si só não indiquenecessariamente um desafio para se alimentar, quando mal adaptadas elas podem retardar a taxa de mastigação, e contribuir ou exacerbar a dificuldade para se alimentar ${ }^{(12)}$. A perda de dentes e a má higiene oral também podem causar mudanças na sensibilidade, dor e prejuízo na mastigação ${ }^{(9)}$, embora grande parte das edêntulas estudadas tenha dieta semi-sólida, demonstrando sua adaptação às condições dentárias.

A prevalência de alterações da deglutição neste estudo (23\%) se mostrou de acordo com uma pesquisa revisada ${ }^{(9)}$, mas significantemente inferior à outra ${ }^{(12)}$, em que a prevalência foi de $68 \%$. Sua associação maior com maior número de diagnósticos de demência, AVE e dependência alimentar está de acordo com a literatura ${ }^{(28)}$.

Os achados desta pesquisa reforçam a necessidade de se gerenciar a deglutição de idosos institucionalizados, visto que fatores como perda da habilidade de se alimentar independentemente e problemas de deglutição são apontados como causa crescente de incapacidade, fator de risco para pneumonia por aspiração, subnutrição, desidratação, asfixia e até morte nos casos mais extremos ${ }^{(26,29)}$.

Nas poucas ILPIs em que o fonoaudiólogo está presente, no Brasil ${ }^{(30)}$, fica clara sua responsabilidade e da equipe multidisciplinar de saúde, pela avaliação e controle de problemas relacionados à alimentação ${ }^{(13)}$. Essa responsabilidade também deve ser atribuída aos cuidadores formais, que muitas vezes prestam assistência inadequada no momento da refeição, por desconhecerem ações apropriadas, o que põe em risco a saúde desses indivíduos ${ }^{(12)}$.

Foram limitações do estudo a não-incorporação de exames objetivos da deglutição, pelas dificuldades estruturais do local onde foi desenvolvido, a não-inclusão de indivíduos do sexo masculino, pelas razões já apresentadas, e a não realização de repetição da filmagem em pelo menos $20 \%$ da amostra, que, entretanto, não pôde ser evitada, devido às características da amostra estudada, sobretudo a natureza progressiva do diagnóstico médico da maioria das residentes e a variação na escala dos cuidadores que ofertavam alimento a cada uma delas. Estudos futuros deverão incluir idosos institucionalizados de ambos os sexos, bem como incorporar exames objetivos.

Esse estudo representa contribuição importante para a identificação de fatores que podem ter impacto na segurança e nutrição de idosos. Comportamentos, atitudes e acomodação de ambientes de alimentação refletem concepções, crenças e valores culturais, daí o interesse de se observar populações de países que ainda estão se adaptando ao desafio de cuidar de seus idosos.

\section{CONCLUSÃO}

Foram verificados nesta amostravários fatores ambientais, cognitivos e comportamentais ligados à alimentação que podem se configurar como risco à deglutição. Também se identificaram outras alterações da deglutição frequentemente associadas à dependência alimentar e alteração cognitiva, especialmente demência, cujo gerenciamento é importante para evitar complicações do estado de saúde destas idosas em situação de fragilidade.

\section{AGRADECIMENTOS}

A Helen Arruda Guimarães, Ana Elizabeth dos Santos Lins, Ana Paula Cajaseiras de Carvalho e Janaína Pivetta Roque pelas valiosas contribuições científicas a este trabalho.

\begin{abstract}
Purpose: To describe the feeding dynamics of institutionalized elderly women regarding the clinical aspects of deglutition, cognition, behavioral and environmental factors related to feeding. Methods: It was carried out a prospective observational cross-sectional study of the feeding dynamics of 30 elderly women, residents at a long-stay nursing home. Some of the evaluated aspects were recorded by the observers at the time of feeding, and others by the analysis of the videotaped register of their mealtime. Data were descriptively presented and compared to literature findings. Results: Mean age was 83.7 years. The most prevalent medical diagnoses were dementia and systemic arterial hypertension. Poor oral intake was observed in $73.3 \%$ of the subjects, as well as an average time of feeding of 10.1 minutes. Attitudinal alterations were observed in 16.7\%, and behavioral alterations, in $40 \%$ of the elderly. The subjects showed inappropriate positioning in $46.7 \%$ of the meals. Assistance was required by $56,6 \%$ of the women: in $70.6 \%$ of the cases, it was provided, and in $66.6 \%$, it was judged inadequate. Edentulism was verified in $44.4 \%$ of the elderly, of which $46.1 \%$ used total denture. Inadequacy of denture adaptation, conservation and hygiene status was predominant $(83.4 \%)$. The prevalence of swallowing alterations was $23.3 \%$, with predominance of the following alterations: food residue after deglutition, cough during feeding, and vocal alterations after deglutition. Conclusion: Environmental, cognitive and behavioral factors related to feeding were significantly present among the subjects of the study, putting deglutition at risk. Other specific deglutition difficulties were also identified. Managing these difficulties is important, in order to avoid health complications for elderly women.
\end{abstract}

Keywords: Health of institutionalized elderly; Feeding; Elderly nutrition; Deglutition disorders; Dementia; Aged; Women; Caregivers 


\section{REFERÊNCIAS}

1. Kalache A, Veras RP, Ramos LR. O envelhecimento da população mundial: um desafio novo. Rev Saúde Pública. 1987;21(3):200-10.

2. Organización Panamericana de la Salud. Las condiciones de salud en las Américas. Washington: OPAS; 1994.

3. Brasil. Ministério do Planejamento, Orçamento e Gestão. Fundação Instituto de Geografia e Estatística. Uma Análise das Condições de Vida da População Brasileira. Estudos e Pesquisas Informações Demográficas e Sócio-Econômica. Rio de Janeiro: IBGE. Síntese de Indicadores Sociais, 22; 2007.

4. Chaimowicz F. A saúde dos idosos brasileiros às vésperas do século XXI: problemas, projeções e alternativas. Rev. Saúde Pública. 1997;31(2):184-200.

5. Veras RP. País jovem com cabelos brancos: a saúde do idoso no Brasil. Rio de Janeiro: Relume Dumará; 1994.

6. Brasil. Ministério do Planejamento, Orçamento e Gestão. Fundação Instituto de Geografia e Estatística, Diretoria de Pesquisas, Coordenação de Trabalho e Rendimento. Pesquisa Nacional por Amostra de Domicílios. Rio de Janeiro: IBGE; 2004-2005.

7. Chaimowicz F, Greco DB. Dinâmica da institucionalização de idosos em Belo Horizonte, Brasil. Rev Saúde Pública. 1999;33(5):454-60.

8. Novaes MRV. A busca do consenso sobre as condições de autonomia e dependência de idosos residentes em instituição de longa permanência: uma metodologia de trabalho para a equipe de cuidados [tese]. São Paulo(SP): Faculdade de Saúde Pública,Universidade de São Paulo; 2005. 239p.

9. Langmore SE, Skarupski KA, Park PS, Fries BE. Predictors of aspiration pneumonia in nursing home residents. Dysphagia. 2002;17(4):298-307.

10. Oh E, Weintraub N, Dhanani S. Can we prevent aspiration pneumonia in the nursing home? J Am Med Dir Assoc. 2004;5(3):174-9.

11. Siebens H, Trupe E, Siebens A, Cook F, Anshen S, Hanauer R, Oster G. Correlates and consequences of eating dependency in institutionalized elderly. J Am Geriatr Soc. 1986;34(3):192-8.

12. Steele CM, Greenwood C, Ens I, Robertson C, Seidman-Carlson R. Mealtime difficulties in a home for the aged: not just dysphagia. Dysphagia. 1997;12(1):43-50; discussion 51.

13. O'Loughlin G, Shanley, C. Swallowing problems in the nursing hame: A novel training response. Dysphagia. 1998;13(3):172-83.

14. Brasil. Poder Executivo. Agência Nacional de Vigilância Sanitária ANVISA. Resolução da Diretoria Colegiada - RDC No. 283 de 26 de setembro de 2005.Aprova o Regulamento Técnico que define normas de funcionamento para as Instituições de Longa Permanência para Idosos. Diário Oficial da União.2005 Set 27.
15. Katz S, Downs TD, Cash HR, Grotz RC. Progress in development of the index of ADL. Gerontologist.1970 Spring;10(1):20-30.

16. Padovani AR, Moraes DP, Mangili LD, Andrade CRF. Protocolo Fonoaudiológico de Avaliação do Risco para Disfagia (PARD). Rev Soc Bras Fonoaudiol. 2007;12(3):199-205.

17. Davim RMB, Torres GV, Dantas SMM. Estudo com idosos de instituições asilares no município de Natal/RN: características socioeconômicas e de saúde. Rev Latinoam Enferm. 2004;12(3):518-24.

18. United States of America. National Institute on Aging. U.S. Department of Commerce. Aging in the United States: past, present and future. Washington (DC); 1998.

19. Marin MJS, Angerami ELS. Caracterização de um grupo de idosas hospitalizadas e seus cuidadores visando o cuidado pósalta hospitalar. Rev Esc Enferm USP. 2002;36(1):33-41.

20. Marrie TJ. Community-acquired pneumonia in the elderly. Clin Infect Dis. 2000;31(4):1066-78. Epub 2000 Oct 20.

21. Langmore SE, Terpenning MS, Schork A, Chen Y, Murray JT, Lopatin D, et al. Predictors of aspiration pneumonia: how important is dysphagia? Dysphagia. 1998;13(2):69-81.

22. Chouinard J, Lavigne E, Villeneuve C. Weight loss, dysphagia, and outcome in advanced dementia. Dysphagia. 1998;13(3):151-5.

23. Dias AB, Zillmer JGV, Fagundes RF, Michels MA. Perfil do idoso morador de instituições de longa permanencia da cidade de pelotas: estudo preliminar. Universidade Federal de Pelotas: XV Congresso de Iniciação Científica, 2006.

24. Kottke FJ. Historia obscura hemiplegiae. Arch Phys Med Rehabil. 1974;55(1):4-13.

25. Simmons SF, Schnelle JF. Feeding assistance needs of long-stay nursing home residents and staff time to provide care. J Am Geriatr Soc. 2006;54(6):919-24.

26. Lee A, Sitoh YY, Lieu PK, Phua SY, Chin JJ. Swallowing impairment and feeding dependency in the hospitalised elderly. Ann Acad Med Singapore. 1999;28(3):371-6.

27. Leopold NA, Kagel MC. Dysphagia - ingestion or deglutition?: a proposed paradigm. Dysphagia. 1997;12(4):202-6.

28. Sonies BC. Oropharyngeal dysphagia in the elderly. Clin Geriatr Med. 1992;8(3):569-77.

29. Kayser-Jones J, Pengilly K. Dysphagia among nursing home residents. Geriatr Nurs.1999;20(2):77-82; quiz 84.

30. Oliveira CA, Silva JOR, Di Ninno CQMS. Intervenção fonoaudiológica nos distúrbios da motricidade oral em instituições que abrigam idosos em Belo Horizonte, MG. Fono Atual. 2006;8(35):41-8. 